\title{
HIGH PRECISION SC CAVITY ALIGNMENT MEASUREMENTS WITH HIGHER ORDER MODES
}

\author{
Stephen Molloy\#, Josef Frisch, Linda Hendrickson, Douglas McCormick, Justin May, Marc Ross, \\ Tonee Smith, SLAC, Stanford, California, USA \\ Nathan Eddy, Sergei Nagaitsev, FNAL, Batavia Illinois USA \\ Nicoleta Baboi, Olaf Hensler, Lyudvig Petrosyan, DESY, Hamburg, Germany \\ Olivier Napoly, Rita Paparella, Claire Simon, CEA, DSM/DAPNIA, Gif sur Yvette, France
}

\section{Abstract}

Experiments at the FLASH linac at DESY have demonstrated that the higher order modes (HOMs) induced in superconducting cavities can be used to provide a variety of beam and cavity diagnostics. The centres of the cavities can be determined from the beam orbit which produces minimum power in the dipole HOM modes. The phase and amplitude of the dipole modes can be used as a high resolution beam position monitor.

For most superconducting accelerators, the existing HOM couplers provide the necessary signals, and the downmix and digitising electronics are straightforward, similar to those for a conventional BPM.

\section{HIGHER ORDER MODES IN SC ACCELERATOR CAVITIES}

In addition to the fundamental accelerating mode, superconducting (SC) cavities support a spectrum of higher order modes (HOMs)[1]. While HOMs can be a source of a variety of accelerator problems (e.g. beam breakup, heating, etc.), they can also be used as beam and cavity diagnostics.

\section{Mode Coupling to the Beam}

HOMs can be characterized by their azimuthal dependence as monopole, dipole, or higher multipole modes. Here we consider the response of these modes to a single electron bunch propagating near the axis of the cavity, and we assume bunches with lengths short compared with the wavelength of the HOM modes.

The interaction of an electron beam with a particular mode is characterised by the loss factor, $k^{(m)}$, or by $R^{(m)} / Q$, whose values may be calculated from numerically calculated fields[2]. The integer, $m$, indicates the order of the mode, with $m=0$ indicating a monopole mode, $m=1$ a dipole mode, etc. The loss factor and $R^{(m)} / Q$ are defined as follows,

$$
\begin{aligned}
& k^{(m)}(r) \equiv \frac{\left|V_{L}^{(m) 2}\right|}{4 \mathrm{U}^{(m)}} \\
& \frac{R^{(m)}}{Q} \equiv \frac{1}{r^{2 \mathrm{~m}}} \cdot \frac{2 \mathrm{k}^{(m)}(r)}{\omega}
\end{aligned}
$$

where $V_{L}^{(m)}$ is the longitudinal potential of the mode (of order $m$ ) at a radius, $r, U^{(m)}$ is the stored energy of the mode, and $\omega$ is its central frequency. The longitudinal potential induced by the beam for each mode will depend on the magnitude of the longitudinal component of the electric field, $E_{z}$.

Since the longitudinal electric field due to the monopole mode has no first order variation with $r$, equation 1 implies that the coupling to the beam does not depend on its offset from the mode axis. The amplitude and phase of monopole modes will, therefore, be governed only by the charge of the bunch, and its time of arrival in the cavity.

The amplitude of the longitudinal electric field of a dipole mode varies in proportion to $r$, so equation 1 implies that the energy coupled into a mode is proportional to $r^{2}$. The amplitude of the potential of a dipole mode will, therefore, be directly proportional to $r$.

This argument may be easily extended to determine the $r$ dependence of quadrupole and higher modes.

Since strongly coupled dipole modes are excited by a beam traversing the cavity with an offset, they may also be excited by a beam entering the cavity with a positive offset, but leaving with a negative offset (i.e. a tilted trajectory that brings it through the centre of the cavity). A tilted bunch will also cause the same effect, and will result in a signal that is $90^{\circ}$ out of phase with the offset signal. A derivation of this may be found in [3]. Dipole modes are therefore excited with an amplitude proportional to bunch charge, and to:

- Transverse position relative to the cavity axis

- Transverse angle relative to the cavity axis. This signal is excited at $90^{\circ}$ phase relative to the position signal.

- Bunch tilt, with amplitude proportional to the bunch length, and $90^{\circ}$ phase relative to the position signal, however, for the short bunch length in FLASH this signal is not significant.

Dipole modes occur in doublets with orthogonal polarisations corresponding to two orthogonal transverse degrees of freedom. The frequencies of these polarisations may be degenerate, or may be split due to asymmetries caused be the cavity couplers and fabrication imperfections.

\footnotetext{
\#smolloy@slac.stanford.edu
} 


\section{HOMs as Diagnostics for FLASH}

A calibration of the dipole HOM amplitude and phase against the beam trajectory could be used to make a HOM based beam position monitor (BPM), and to calculate the trajectory that results in the lowest HOM power. This trajectory defines the axis of the mode under investigation, and a comparison of the axes calculated for different cavities in an accelerating module is a measure of their relative alignment. It should be noted that this is a measure of the relative alignment of the centre of one mode only, whose correlation to the centre of the fundamental mode is not known. Future measurements are planned to investigate this.

In most superconducting linacs, including FLASH, special couplers are used to damp the HOM modes, thereby preventing beam instabilities. The HOM signals are transported outside of the tunnel and are thus available for use without requiring any additional beam line components.

The FLASH cavity HOM frequencies were simulated[2], and have been measured[4]. Of particular interest for beam position measurement and cavity alignment diagnostics are the first two dipole bands at $\sim 1.6$ and $\sim 1.9 \mathrm{GHz}$.

The dipole mode TE111-6 at $\sim 1.7 \mathrm{GHz}$ was used for these measurements. This mode has a relatively high calculated $R / Q$ of $5.5 \mathrm{Ohms} / \mathrm{cm}^{2}$.

\section{HOM MEASUREMENT SYSTEM}

A narrowband (20 MHz) system, centred at the TE1116 frequency of $1.7 \mathrm{GHz}$, was used for the beam position measurements. This system down-mixed the HOM signal to an intermediate frequency (IF) of approximately 20 $\mathrm{MHz}$, which was then digitized by a 14 bit, $108 \mathrm{MS} / \mathrm{s}^{1}$ digitiser. A schematic of this system is shown in figure 1.

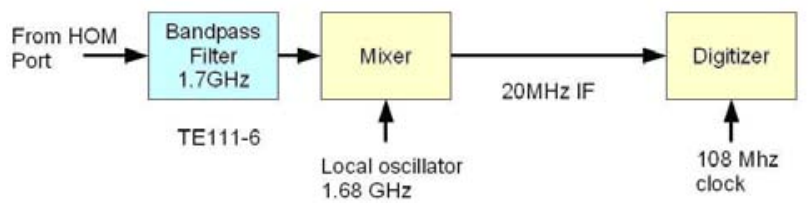

Figure 1: Block diagram of the TE111-6 narrowband measurement electronics.

It should be noted that an additional $\sim 1.7 \mathrm{GHz}$ signal (known as the calibration tone) was added to the signal before mixing. This was originally intended to provide a means of automatic calibration, however it was never used for this purpose. This signal was always present during the data collection periods.

The local oscillator (LO), calibration tone, and digitiser clocks were all phase locked with the accelerating RF.

A more detailed description of the electronics may be found in [5].

\footnotetext{
${ }^{1}$ MegaSamples per second.
}

\section{DIPOLE MODES FOR BEAM ORBIT MEASUREMENT}

Cavity BPMs can provide resolutions better than 20 $\mathrm{nm}[6]$. HOM dipole modes can be used in a fashion similar to cavity BPMs, although at poorer resolution as the accelerating cavities are not optimized for this purpose. The HOM measurements have the advantage that the majority of the linac length is occupied by accelerator structures.

Measurements based on HOMs have the additional advantage that they measure the displacement of the beam from the centre of a strongly coupled, and potentially damaging, dipole mode, whereas the centre of BPM based measurements is defined by the misalignment of the BPMs with respect to the cavity. This is true even in the case where the HOM measurements are calibrated using the BPMs, since the difference between the centres will be a simple offset term that can be subtracted.

\section{Dipole Mode Signals}

Each of the dipole modes contains two lines with a splitting, in the case of the FLASH linac, comparable to the line width. Some typical dipole signals are shown in figure 2 .
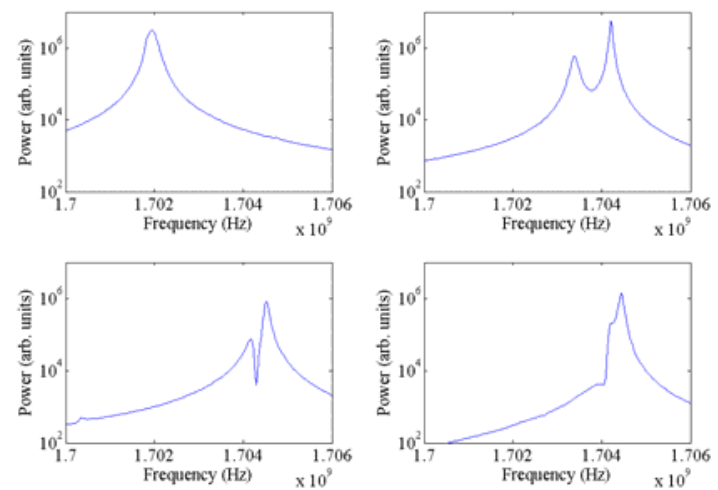

Figure 2: HOM dipole spectra for different cavities showing splitting of polarizations.

It has proven difficult to fit to these signals to obtain the frequencies and decay times of the two polarisations in the dipole mode. Instead Singular Value Decomposition (SVD) is used to find an orthonormal basis for the data sets.

SVD decomposes a matrix, $X$, into the product of three matrices as follows[7],

$$
X=U \cdot S \cdot V^{T}
$$

where $U$ and $V$ are unitary, $S$ is diagonal, and $V^{T}$ indicates the transpose of $V$. Due to the unitary nature of $U$ and $V$ the following follows from equation 3 ,

$$
X^{T} \cdot X=V \cdot S^{2} \cdot V^{T}
$$

Thus, in the case where $X$ is a $i x j$ matrix containing the data from $i$ beam pulses with $j$ digitiser samples recorded for each pulse, $V$ is the matrix of eigenvectors of the cross-correlation matrix of $X$, and the diagonal elements 
of $S^{2}$ are the eigenvalues. The eigenvectors given by $V$ are referred to as the "modes" of the data, since linear combinations of these vectors provide a close fit to the output of the electronics for each beam pulse. It can be shown[7] that, in the case where the strongest $k$ modes are used to represent the data, the 2-norm distance between the reconstructed matrix and the measured matrix will be equal to the $(k+1)$ th eigenvalue. Due to the four transverse degrees of freedom of the beam, it is important to use at least four SVD modes (i.e. $k \geq 4$ ). Typically six modes are used. Since the difference between the first and sixth largest eigenvalues has been measured[8] to be greater than two orders of magnitude, it can be concluded that these provide a sufficiently good representation of the data.

Note that these modes will be linear combinations of the intuitive cavity modes. Also, since the SVD will pick the largest modes irrespective of the frequency and $Q$ of the actual cavity modes, its success is not dependant on the degree of degeneracy of the orthogonal polarisations.

The amplitudes of the strongest SVD modes are measured for each machine cycle by finding the value of its dot product with the data. Linear regression is then used to correlate these mode amplitudes with the beam position measured by conventional BPMs.

This method, including the data preparation, SVD, and linear regression, is described in more detail in [8].

\section{Beam Position Measurement Results}

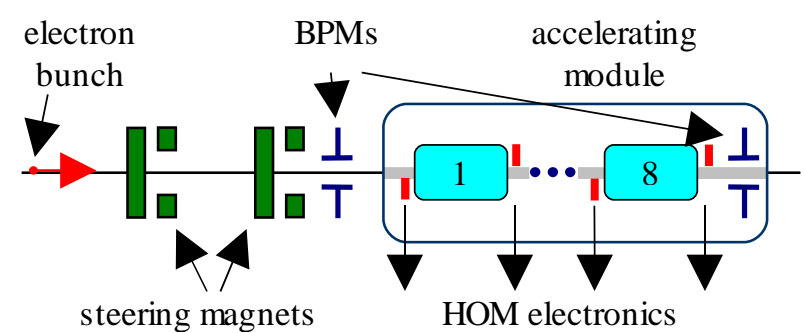

Figure 3: Steering setup for HOM dipole experiment.

The beam was steered over a range of approximately 5 $\mathrm{mm}$ in $\mathrm{X}$ and $\mathrm{Y}$, and approximately $10 \mathrm{~m} . \mathrm{rad}$ in $\mathrm{X}^{\prime}$ and $Y^{\prime}$. The HOM mode amplitudes were calibrated relative to the positions interpolated from BPMs. Due to the presence of RF focussing, coupler kicks, etc., this will introduce systematic errors, however, in the case of the high energy modules (i.e. ignoring ACC1), these should be negligible. In order to eliminate spurious correlations with position and angle from the calculations, each of the four dimensional points were chosen randomly. The experimental configuration is shown in figure 3 .

Pulses with saturated HOM signals, low beam current, or bad orbits as measured by the conventional BPMs were rejected. The position measured by the fifth cavity ${ }^{2}$ of the accelerator module (containing eight cavities) was compared with the positions predicted by the first and last

\footnotetext{
${ }^{2}$ The choice of cavity for this measurement was quite arbitrary, so cavity 5 was chosen due to its proximity to the centre of the module.
}

cavities in the module as a measurement of the HOM position resolution. The spread of the residuals in $\mathrm{X}, \mathrm{X}$, $\mathrm{Y}$, and $\mathrm{Y}^{\prime}$ is shown in figures $4-7$.

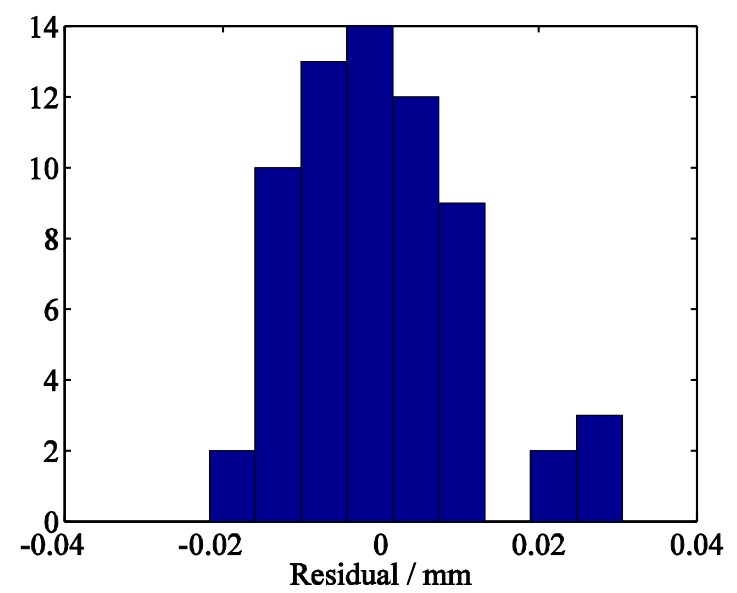

Figure 4: Spread of residuals in the X measurement.

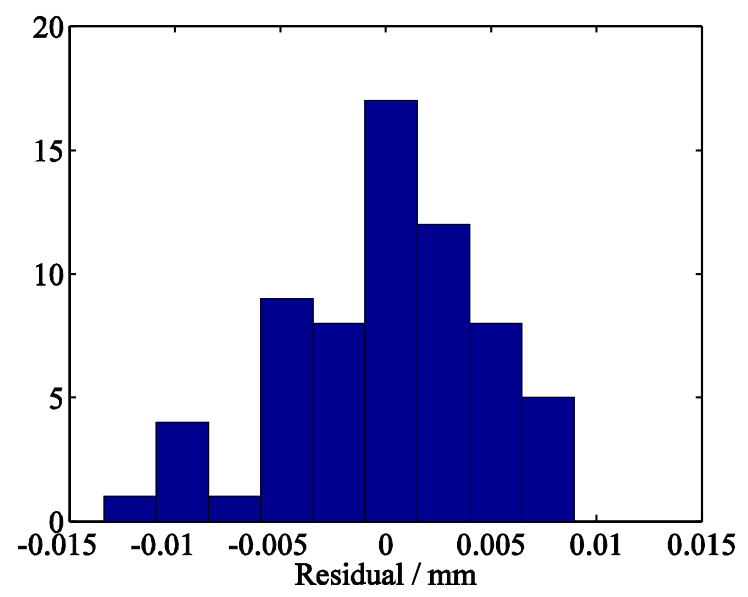

Figure 5: Spread of residuals in the Y measurement.

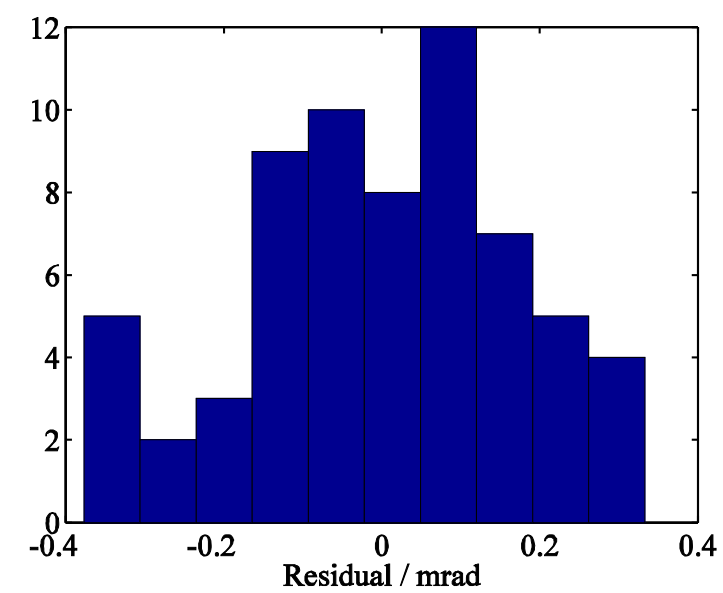

Figure 6: Spread of residual in the X' measurement.

A position resolution of 9 microns in $\mathrm{X}, 4$ microns in $\mathrm{Y}$, and an angular resolution of 175 micro.rad in $X^{\prime}$, and 140 micro.rad in $Y^{\prime}$, has been measured.

This is in agreement with the theoretical value once imperfections in the electronics, and the uncertainty in the charge used for normalization of the HOM amplitudes 
has been taken into account [9]. It is also consistent with subsequent resolution measurements from other cavities in different modules.

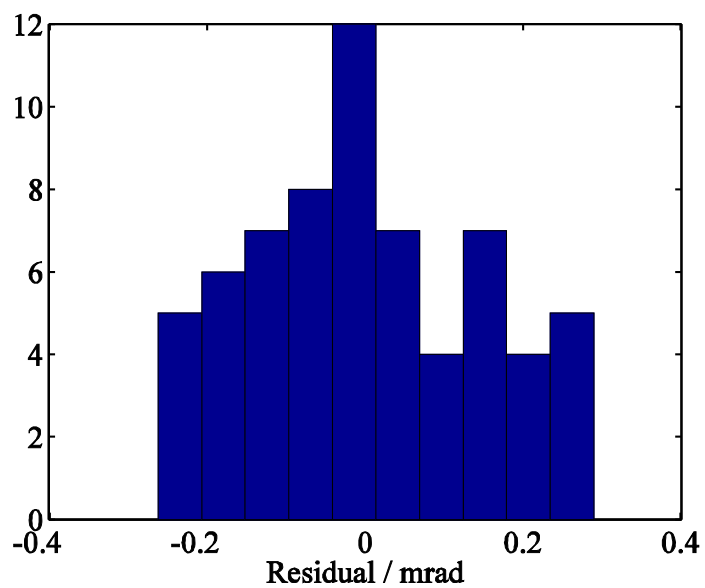

Figure 7: Spread of residuals in the Y' measurement.

\section{CAVITY ALIGNMENT MEASUREMENT}

There exists an orbit through each cavity which will null the HOM power for a particular dipole mode. The beam $\mathrm{X}$ and $\mathrm{Y}$ (but not $\mathrm{X}$ angle and $\mathrm{Y}$ angle) were found which minimized the HOM power in each of the cavities of a structure. The relative positions of these minima for each cavity of the module were used to measure the alignment of the module.

\section{Algorithm to Find Cavity Centers}

As described above, SVD can be used to find the six strongest HOM mode amplitudes. Linear regression can then be used to correlate these amplitudes with beam position. The position corresponding to the minimum of the sum of the squares of these amplitudes can then be calculated.

\section{Cavity Alignment Measurement}

The beam was steered in X, Y, X', and Y' in the fourth and fifth modules (ACC4 and ACC5). Approximately twenty runs, of $\sim 100$ points each were used. The alignments are plotted with the slope and offset removed to show the relative alignment of the cavities within the module, figure 7 and figure 8 .

In ACC4, the RMS alignment in $\mathrm{X}$ and $\mathrm{Y}$ was 105 and 215 microns, with a measurement reproducibility in $\mathrm{X}$ and $\mathrm{Y}$ of 37 and 24 microns.

In ACC5, the RMS alignment in X and $\mathrm{Y}$ was 241 and 203 microns, with a measurement reproducibility of 9 and 5 microns. The specification for cavity alignment is 300 microns RMS.

It is important to note that this measurement does not measure the cavity alignment directly, but only measures the relative positions of the axes of the TE111-6 dipole modes in the cavities in a module. The dipole mode axes may not be aligned to the mechanical centres of the cavities due to perturbations from couplers, and manufacturing imperfections. If the dominant influence to the shifting of the centre of this mode is the location of the couplers, then this can be expected to be a good indication of the cavity alignment since the coupler placement is identical for each cavity inside a module. If, on the other hand, manufacturing errors dominate the location of this mode, then this measurement may contain no knowledge on the relative alignment within the module. The question of the dominate source of this centre shift has yet to be answered, however it should be noted that the alignment results shown in figures 8 and 9, do not correlate well with the results obtained from the wire position monitors[10].
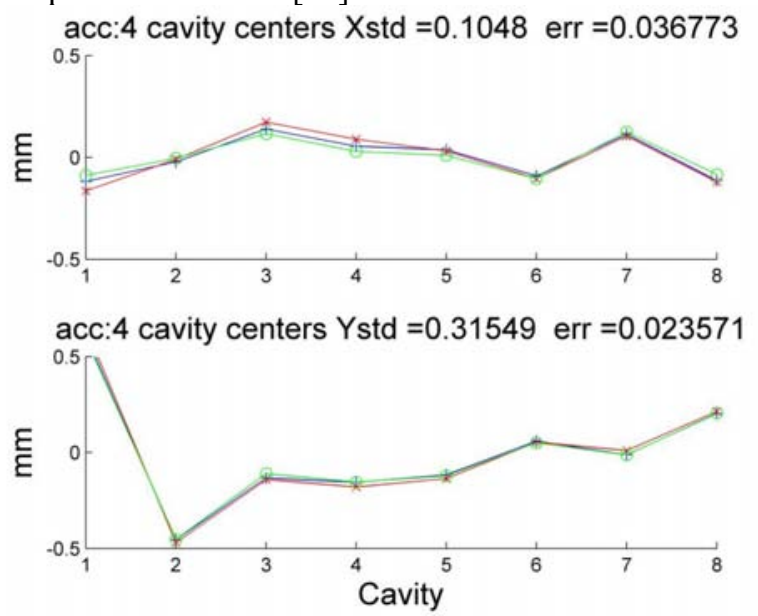

Figure 8: Alignment of TE111-6 cavity mode centres in module 4.

acc $: 5$ cavity centers Xstd $=0.24111$ err $=0.0089929$
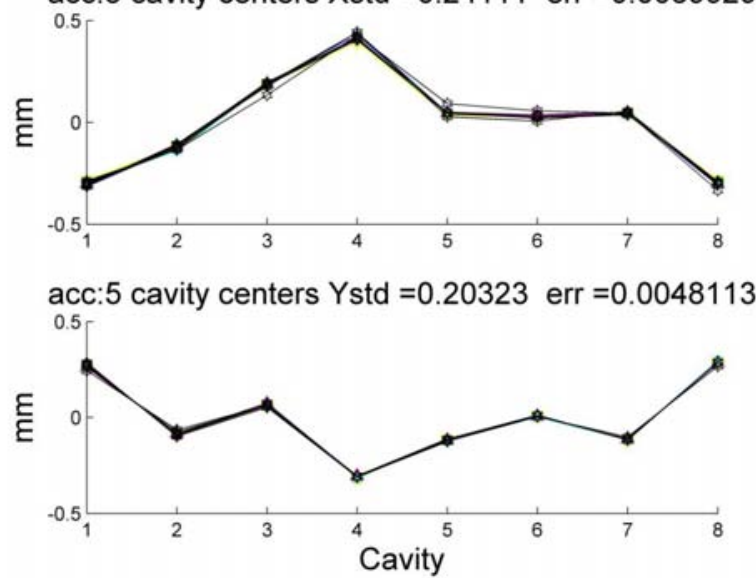

Figure 9: Alignment of TE111-6 cavity modes centres in module 5 .

\section{CONCLUSIONS}

Since the lengths of both the ILC and the XFEL are dominated by cavities, HOM based BPMs would give the ability to monitor the position of the beam throughout the majority of both these machines.

An important benefit of measuring the beam position using HOMs is that the position reported is with respect to the HOM center. A simple feedback algorithm could be developed to maintain the orbit at a location that results in the lowest HOM power output, thus minimising the 
negative affects of the HOMs; increased cryogenic load, beam break-up, etc.

A HOM based BPM system has been developed at FLASH, and shown to have a position resolution of $<10$ microns. It was also shown how the alignment of a strongly coupled dipole mode could be measured with the same resolution. The alignment of this mode within two of the TTF modules (ACC4 and ACC5) was shown to be less than 300 microns, which, if the dipole mode alignment is an indication of the fundamental mode alignment, is the specified tolerance for these modules.

\section{ACKNOWLEDGMENTS}

We appreciate the support of the FLASH crew during our measurements. In spite of technical difficulties with the facility, we managed with their help, to finish our experimental program in optimum conditions. This work was supported by the U.S. Department of Energy Contract No. DE-AC02-76SF00515, and by the European Community FP6 "Structuring the European Research Area" program (CARE, Contract No. RII3-CT-2003506395).

\section{REFERENCES}

1: T.Weiland, R.Wanzenburg, Wake Fields and Impedances, 1990, US-CERN Accelerator Course

2: R.Wanzenberg, Monopole, Dipole, and Quadrupole Passbands of the TESLA 9-cell cavity, 2001, TESLA report 2001-33

3: M. Ross et al., RF Cavity BPMs as Beam Angle and Beam Correlation Monitors, 2003, PAC03, Portland, Oregon

4: G. Kreps, Private Communication

5: J. Frisch et al, Electronics and Algorithms for HOM Based Beam Diagnostics, 2006, Beam Instrumentation Workshop, Fermilab
6: S. Walston et al, Performance of a Nanometer Resolution BPM System, 2006, EPAC, Edinburgh, Scotland

7: G.H. Golub and C.F. Van Loan, Matrix Computations, 1996

8: S. Molloy et al, High precision superconducting cavity diagnostics with HOM measurements., 2006, PRST-AB, 9, 11

9: J. Frisch et al, High Precision SC Cavity Diagnostics with HOM Measurements, 2006, EPAC, Edinburgh, Scotland

10: J. Frisch, Private Communication 